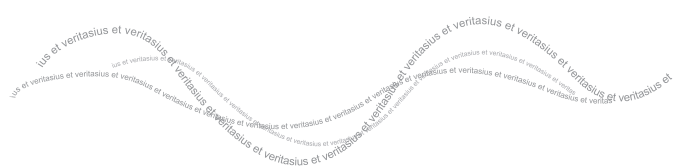

\title{
Los servicios públicos de telecomunicaciones y las exigencias ambientales para la instalación de su infraestructura(*)
}

\section{Public telecommunication services and the environmental requirements for the development of its infrastructure}

\author{
Cyndel Caballero Fernández ${ }^{(*)}$ \\ Rodrigo, Elías \& Medrano Abogados (Lima, Perú) \\ Diego Morales Alferrano ${ }^{(*+*)}$ \\ Rodrigo, Elías \& Medrano Abogados (Lima, Perú)
}

\begin{abstract}
Resumen: El presente artículo describe las principales obligaciones y exigencias ambientales para la implementación de la infraestructura necesaria para la prestación de servicios públicos de telecomunicaciones tales como la obtención de instrumentos de gestión ambiental, el cumplimiento de los Límites Máximos Permisibles (LMP) y la observancia de principios ambientales. Lo anterior con la finalidad de resaltar que, conforme al marco legal aplicable, las medidas antes señaladas tienen como objetivo que la ejecución de esta actividad económica no genere impactos negativos significativos en el medio ambiente, los recursos naturales y la salud de las personas.
\end{abstract}

Palabras clave: Infraestructura de telecomunicaciones - Servicios públicos de telecomunicaciones - Obligaciones ambientales - Principio precautorio - Límites máximos permisibles - Radiaciones no ionizantes

\begin{abstract}
This article describes the main environmental obligations for the implementation of infrastructure for the provision of public telecommunication services like the obtaining of environmental management instruments, the compliance of maximum permissible limits and environmental principles. The purpose of the referred measures is to prevent that this activity causes significant negative impacts on the environment, natural resources, and people's health.
\end{abstract}

Keywords: Telecommunications infrastructure - Public telecommunications services - Environmental liabilities - Precautionary principle - Maximum permissible limits - Nonionizing radiation

$\left({ }^{*}\right) \quad$ Nota del Equipo Editorial: Este artículo fue recibido el 01 de agosto de 2021 y su publicación fue aprobada el 26 de noviembre de 2021.

(**) Abogada por la Pontificia Universidad Católica del Perú. Asociada de Rodrigo, Elías \& Medrano Abogados. ORCID: https://orcid. org/0000-0002-2176-1904. Correo electrónico: dcaballero@estudiorodrigo.com.

${ }^{* * *}$ Abogado por la Pontificia Universidad Católica del Perú. Asociado de Rodrigo, Elías \& Medrano Abogados. ORCID: https://orcid. org/0000-0002-5336-5324. Correo electrónico: dmorales@estudiorodrigo.com. 


\section{Introducción}

Los titulares de servicios de telecomunicaciones se encuentran obligados a realizar inversiones en infraestructura, con la finalidad de que estos servicios puedan ser brindados en condiciones de calidad y eficiencia a la población. Sin embargo, en el marco de la instalación y la operación de estas infraestructuras es necesario cumplir con ciertas disposiciones y procedimientos establecidos en el ordenamiento legal, que tienen como objetivo, entre otros, salvaguardar determinados bienes jurídicos.

Así, por ejemplo, la normativa sectorial impone una serie de deberes a los titulares de infraestructuras de telecomunicaciones relacionados a la protección del ornato, el patrimonio cultural de la nación, la salud pública, las áreas naturales protegidas y, en particular, el medio ambiente. Siendo ello así, el presente artículo tiene como finalidad revisar y analizar las principales obligaciones y exigencias ambientales relacionadas a la implementación de infraestructuras para la prestación de servicios públicos de telecomunicaciones. Asimismo, evidenciar ciertos vacíos legales en materia ambiental en relación con el desarrollo de este tipo de instalaciones como, por ejemplo, la inexistencia de un reglamento de protección ambiental en el sector.

Para ello, revisaremos los principales dispositivos legales que regulan esta actividad; es decir, la Ley para el Fortalecimiento de la Expansión de Infraestructura en Telecomunicaciones, aprobada por Ley 29022, y sus reglamentos, aprobados por el Decreto Supremo 039-2007MTC (“D.S. 039-2007") y el Decreto Supremo 003-2015MTC (“D.S. 003-2015"). En particular, nuestro análisis se centrará en los siguientes aspectos: (i) la aplicación del Principio Precautorio; (ii) la regulación de los Límites Máximos Permisibles ("LMP") de Radiaciones No lonizantes ("RNI"); (iii) los Instrumentos de Gestión Ambiental ("IGA") exigibles para la instalación de infraestructura de telecomunicaciones; y, (iv) la aprobación de un Reglamento de Protección Ambiental para el sector telecomunicaciones.

\section{Conceptos generales}

\subsection{Los servicios públicos de telecomunicaciones y su infraestructura \\ De conformidad con lo establecido en la legislación}

nacional, las telecomunicaciones son toda transmisión y/o emisión y recepción de señales que represente signos, escrituras, imágenes, sonidos o información de cualquier naturaleza por medios físicos, medios electromagnéticos, medios ópticos u otros ${ }^{(1)}$. El Texto Único Ordenado de la Ley de Telecomunicaciones, aprobado por el Decreto Supremo 013-93-TCC ("Ley de Telecomunicaciones"), y su Reglamento General, cuyo Texto Único Ordenado fue aprobado mediante el Decreto Supremo 0202007-MTC ("Reglamento General"), señalan que los servicios de telecomunicaciones pueden clasificarse ${ }^{(2)}$ sobre la base de dos criterios:

a) Según su operación, se clasifican en servicios portadores, teleservicios (o servicios finales), servicios de difusión y servicios de valor añadido; $y$,

b) De acuerdo a su utilización y naturaleza, se clasifican en servicios públicos, servicios privados y servicios privados de interés público (énfasis agregado).

Los servicios públicos de telecomunicaciones son aquellos que se encuentran a disposición de la población en general, a cambio del pago de una contraprestación tarifaria por su utilización ${ }^{(3)}$. Los interesados en la prestación de estos servicios deben obtener, previamente, una concesión única que será otorgada por el Ministerio de Transportes y Comunicaciones ("MTC") en observancia de las disposiciones y el procedimiento regulado en la Ley de Telecomunicaciones y su Reglamento General.

Una vez obtenido dicho título habilitante, los operadores tienen que cumplir con determinadas obligaciones establecidas en el marco legal, entre ellas, instalar infraestructura que les permita brindar los servicios que les han sido otorgados en

(1) Definición recogida en el Anexo del Reglamento General.

(2) Véase los artículos 8 y 9 de la Ley de Telecomunicaciones, así como los artículos 21 y 22 del Reglamento General.

(3) Al respecto, el artículo 40 de la Ley de Telecomunicaciones señala lo siguiente: "Serán considerados servicios públicos de telecomunicaciones aquellos servicios declarados como tales en el reglamento de esta Ley, que estén a disposición del público en general y cuya utilización se efectúe a cambio del pago de una contraprestación. Su prestación será normada por la presente Ley y podrá ser reglamentada cuando por las características del servicio ello fuere necesario. Los servicios portadores serán considerados necesariamente servicios públicos". 
concesión ${ }^{(4)}$. Sin embargo, a inicios del presente siglo, se advirtió que los procedimientos destinados al otorgamiento de autorizaciones para la instalación de infraestructura de telecomunicaciones se veían dilatados o paralizados debido a las diversas trabas impuestas por las autoridades administrativas (especialmente, en el caso de los gobiernos locales). Así, por ejemplo, Diego Zegarra destacó lo siguiente: "(...) en los últimos años se han identificado una serie de prácticas vinculadas con la actuación de Municipalidades que se han convertido en verdaderos factores de limitación de desarrollo de las infraestructuras (...)" (2008, p. 323).

En este contexto, considerando que los servicios públicos de telecomunicaciones son de interés y necesidad pública para el desarrollo social y económico del país, el Estado promulgó la Ley 29022, mediante la cual se instauró un régimen especial y temporal para la implementación de la infraestructura necesaria para la prestación de los servicios en cuestión. Esta norma, entre otros, tiene como objetivo estandarizar el procedimiento para la obtención de los títulos habilitantes requeridos para la instalación de este tipo de infraestructuras, por lo que se indicó que sus disposiciones son de observancia obligatoria para todas las entidades de la Administración Pública. De esa manera, el Estado buscó otorgar mayor predictibilidad a los administrados, a fin de promover la inversión privada en el desarrollo de las telecomunicaciones.

La Ley 29022 entró en vigencia al día siguiente de la publicación de su primer reglamento, aprobado por el D.S. 039-2007. En la actualidad, este último ha sido derogado casi en su integridad por el D.S. 003-2015, que aprobó el nuevo reglamento de esta ley(5). EI D.S. 003-2015 establece, entre otros, los requisitos que deben presentar los interesados(6) para obtener las autorizaciones requeridas para la instalación de infraestructuras de telecomunicaciones. Sobre el particular, y considerando los objetivos del presente artículo, resulta importante destacar que el D.S. 003-2015, a diferencia de lo que ocurría con el D.S. 039-2007, exige que para el otorgamiento de estos títulos habilitantes sea indispensable la presentación de un IGA aprobado por el MTC.
Como explicaremos en los siguientes párrafos, actualmente, los titulares de proyectos de inversión en infraestructura de telecomunicaciones deben tener en cuenta el cumplimiento de ciertas obligaciones ambientales: (i) antes del desarrollo de la actividad (la obtención de un IGA); y, (ii) durante su operación (como la observancia de los LMP de RNI, el Principio Precautorio y los Estándares de Calidad Ambiental para RNI).

\subsection{Regulación ambiental aplicable}

La gestión ambiental en el país, tal y como hoy las conocemos, se reguló a partir del año 2005, con la Ley General del Ambiente, aprobada mediante Ley 28611 ("LGA"). Esta norma fue emitida con el objetivo de asegurar el cumplimiento efectivo de, entre otros, el derecho que tiene toda persona a un ambiente saludable, equilibrado y adecuado para el pleno desarrollo de la vida ${ }^{(7)}$. Así, la LGA creó un marco normativo dirigido a ordenar la gestión ambiental del país, destacando el rol que para ello cumplirían ciertos mecanismos como, por ejemplo, los principios e instrumentos ambientales. Veamos.

\subsubsection{Los principios de la gestión ambiental}

El título preliminar de la LGA desarrolla una serie de principios que constituyen los pilares de la gestión ambiental en el país. Sobre estos, la norma instituye una "jerarquía de mitigación" que el Gobierno debe tomar en cuenta antes de aprobar una política nacional o autorizar una actividad que, por sus características, podría afectar el medio ambiente, los recursos naturales y/o la salud de las personas. Esta jerarquía prioriza el Principio de Prevención por sobre los demás principios regulados en la LGA. Así, se determina que, en la gestión ambiental,

(4) El numeral 2 del artículo 130 del Reglamento General establece que es obligación de los concesionarios "instalar la infraestructura que se requiera para la prestación del servicio que se otorga en concesión, cumpliendo las normas municipales o de otros organismos públicos, las cuales no podrán constituir barreras de acceso al mercado".

(5) A la fecha, se encuentran vigentes el artículo 19 (solicitud de facilidades ante la empresa concesionaria de servicios públicos) y el Capítulo I del Título III (Del otorgamiento de facilidades por la Concesionaria de infraestructura de Carreteras) del D.S. 039-2007.

(6) Es importante destacar que, conforme al D.S. 003-2015, las disposiciones del régimen especial son de aplicación tanto para los operadores de servicios públicos de telecomunicaciones como para los proveedores de infraestructura pasiva. Estos últimos son aquellos que, sin contar con la calidad de concesionarios, se encuentran habilitados para desplegar infraestructura, para lo cual se deberán inscribir previamente en el Registro de Proveedores de Infraestructura Pasiva del MTC.

(7) Artículo 2.- Toda persona tiene derecho:

(...) 22. A la paz, a la tranquilidad, al disfrute del tiempo libre y al descanso, así como a gozar de un ambiente equilibrado y adecuado al desarrollo de su vida (énfasis agregado). 
el Estado siempre está obligado a evitar cualquier tipo de degradación del ambiente, y solo cuando esto no sea posible, se encuentra habilitado para adoptar medidas de mitigación, recuperación, restauración o eventual compensación, según corresponda $^{(8)}$.

En esa línea, la LGA enfatiza que la gestión ambiental también debe guiarse bajo el Principio Precautorio que exige que la falta de certeza absoluta, respecto de un peligro de daño grave o irreversible, no se debe utilizar como razón para postergar la adopción de medidas eficaces y eficientes para impedir la degradación del ambiente ${ }^{(9)}$. Este principio “(...) se origina en la búsqueda de mayor eficacia y eficiencia en la actuación gubernamental para la protección ambiental" (Alegre, 2010 , p. 20), debido a que no siempre será posible identificar los efectos negativos que una actividad podría generar en el ambiente. Así, "[e]n estos casos, opera el principio precautorio, con la finalidad de adoptar tempranamente una decisión que permita evitar un daño grave, aun cuando no haya certeza científica de que se vaya a generar" (Alegre, 2010, p. 21).

Al respecto, resulta importante destacar que este principio ha servido para exigir que ciertas actividades cuyos posibles impactos ambientales no habían sido identificados, debido a la incertidumbre científica, cumplan con medidas razonables y provisionales durante su ejecución. Ello, con la finalidad de evitar o reducir un presunto peligro derivado de realizar dichas actividades sin un parámetro ambiental adecuado. De hecho, el Principio Precautorio ha sido la guía en materia ambiental para la implementación de infraestructura necesaria para la prestación de servicios públicos de telecomunicaciones. Esto último considerando que, antes de que la normativa ambiental incluya expresamente al sector telecomunicaciones (i.e. 2011) dentro de las actividades obligadas a obtener un IGA, el Principio Precautorio fue recogido por la normativa sectorial (Ley 29022 y el D.S. 039 2007) a fin de orientar la ejecución de esta actividad y evitar la generación de impactos negativos significativos en el medio ambiente.

\subsubsection{Los IGA}

Por otro lado, el artículo 16 de la $\operatorname{LGA}^{(10)}$, define a los IGA como aquellos "mecanismos orientados a la ejecución de la política ambiental, sobre la base de los principios establecidos en la presente Ley, y en lo señalado en sus normas complementarias y reglamentarias". Considerando lo anterior, el artículo 17 de la misma norma(11) determina qué mecanismos podrán ser considerados como IGA, precisando que, en general, son todos aquellos orientados al cumplimiento de la Política Nacional Ambiental y las normas ambientales que rigen en el país y que, entre otros, se dirigen a la planificación, promoción, prevención, control, corrección, información, financiamiento, participación y fiscalización ambiental.

Para la instalación y operación de infraestructura de telecomunicaciones, destacan los siguientes IGA: (i) los LMP, como IGA de fiscalización; (ii) los IGA sujetos

(8) Artículo Vl.- Del principio de prevención

La gestión ambiental tiene como objetivos prioritarios prevenir, vigilar y evitar la degradación ambiental. Cuando no sea posible eliminar las causas que la generan, se adoptan las medidas de mitigación, recuperación, restauración o eventual compensación, que correspondan.

(9) Artículo VII del Título Preliminar de la LGA

(10) Artículo 16.- De los instrumentos

16.1 Los instrumentos de gestión ambiental son mecanismos orientados a la ejecución de la política ambiental, sobre la base de los principios establecidos en la presente Ley, y en lo señalado en sus normas complementarias y reglamentarias.

16.2 Constituyen medios operativos que son diseñados, normados y aplicados con carácter funcional o complementario, para efectivizar el cumplimiento de la Política Nacional Ambiental y las normas ambientales que rigen en el país.

(11) Artículo 17.- De los tipos de instrumentos

17.1 Los instrumentos de gestión ambiental podrán ser de planificación, promoción, prevención, control, corrección, información, financiamiento, participación, fiscalización, entre otros, rigiéndose por sus normas legales respectivas y los principios contenidos en la presente Ley.

17.2 Se entiende que constituyen instrumentos de gestión ambiental, los sistemas de gestión ambiental, nacional, sectoriales, regionales o locales; el ordenamiento territorial ambiental; la evaluación del impacto ambiental; los Planes de Cierre; los Planes de Contingencias; los estándares nacionales de calidad ambiental; la certificación ambiental, las garantías ambientales; los sistemas de información ambiental; los instrumentos económicos, la contabilidad ambiental, estrategias, planes y programas de prevención, adecuación, control y remediación; los mecanismos de participación ciudadana; los planes integrales de gestión de residuos; los instrumentos orientados a conservar los recursos naturales; los instrumentos de fiscalización ambiental y sanción; la clasificación de especies, vedas y áreas de protección y conservación; y, en general, todos aquellos orientados al cumplimiento de los objetivos señalados en el artículo precedente.

17.3 El Estado debe asegurar la coherencia y la complementariedad en el diseño y aplicación de los instrumentos de gestión ambiental. 
al Sistema de Evaluación del Impacto Ambiental ("SEIA"), como IGA preventivos; y, (iii) Ios IGA no sujetos al SEIA, como IGA complementarios. A continuación, presentamos una introducción general de estos IGA, sin perjuicio de, posteriormente, desarrollar cuáles son sus implicancias en el sector telecomunicaciones.

\section{El artículo 22 de la LGA ${ }^{(12)}$ define a los LMP como}

La medida de la concentración o grado de elementos, sustancias o parámetros físicos, químicos y biológicos, que caracterizan a un efluente o una emisión, que al ser excedida causa o puede causar daños a la salud, al bienestar humano y al ambiente.

En ese sentido, se instituye a los LMP como un IGA de fiscalización que, en conjunto con los Estándares de Calidad Ambiental ("ECA")(13), tienen como objetivo mejorar la calidad de vida de las personas (De La Puente, 2008, p. 220).

\section{Los LMP:}

Tienen relación directa con los efectos que la actividad del hombre podría generar sobre el medio ambiente, sustentándose en las normas constitucionales que disponen que el ejercicio de la libertad de empresa no debe ser lesivo a la salud y que el derecho de propiedad debe ser ejercido en armonía con el bien común y dentro de los límites de ley (De La Puente, 2008, p. 225).

Así, a través de este instrumento el Gobierno determina "la 'línea' de aceptabilidad o tolerancia de algunos daños ambientales en la vida de la sociedad (...)" (De La Puente, 2008, p. 223).

Por ello, una importante diferencia legal entre los ECA y los LMP es que estos últimos "son exigibles a la industria por la autoridad ambiental competente, mientras que los ECA no, a menos que se demuestre que existe causalidad entre una persona jurídica y la trasgresión de dichos estándares". De manera que, "[I]os LMP son utilizados para el control de las fuentes puntuales de contaminación y están referidos a los valores límites, mientras que los ECA tiene relación con los objetivos de la calidad del cuerpo receptor" (De La Puente, 2008, pp. 225-226).
En el caso del sector telecomunicaciones, previamente a la aprobación de la LGA (i.e. 2005), el MTC aprobó los LMP exigibles para los titulares de este tipo de actividades. Precisamente, y considerando lo dispuesto en el Reglamento Nacional para la Aprobación de ECA y LMP, aprobado por el Decreto Supremo 044-98-PCM, se emitió el Decreto Supremo 038-2003-MTC ("D.S. 0382003") que regula los LMP de RNI en telecomunicaciones, como un IGA dirigido a "prevenir y controlar la contaminación generada por actividades comprendidas en el subsector telecomunicaciones, sobre la base de una estrategia destinada a proteger la salud, mejorar la competitividad del país y promover el desarrollo sostenible"(14).

En ese sentido, los LMP de RNI se constituyeron como el principal IGA del sector telecomunicaciones. Ello, hasta que se efectivizaron las exigencias de la Ley del Sistema Nacional de Evaluación del Impacto Ambiental, aprobada por Ley 27446 ("Ley SEIA"), con la entrada en vigencia de su reglamento, mediante el Decreto Supremo 019-2009-MINAM ("Reglamento SEIA")(15).

La Ley SEIA desarrolla la figura de los IGA en el ordenamiento jurídico peruano, diferenciando entre: (i) Ios IGA sujetos al SEIA; y, (ii) Ios IGA complementarios al SEIA. Los IGA sujetos al SEIA son IGA preventivos, dirigidos a evitar que las actividades económicas generen impactos ambientales negativos significativos en el ambiente. De acuerdo con el artículo 11 del Reglamento SEIA, son tres Ios IGA sujetos

(12) Artículo 32.- Del Límite Máximo Permisible

32.1 El Límite Máximo Permisible - LMP, es la medida de la concentración o grado de elementos, sustancias o parámetros físicos, químicos y biológicos, que caracterizan a un efluente o una emisión, que al ser excedida causa o puede causar daños a la salud, al bienestar humano y al ambiente. Su determinación corresponde al Ministerio del Ambiente. Su cumplimiento es exigible legalmente por el Ministerio del Ambiente y los organismos que conforman el Sistema Nacional de Gestión Ambiental. Los criterios para la determinación de la supervisión y sanción serán establecidos por dicho Ministerio.

32.2 EI LMP guarda coherencia entre el nivel de protección ambiental establecido para una fuente determinada y los niveles generales que se establecen en los ECA. La implementación de estos instrumentos debe asegurar que no se exceda la capacidad de carga de los ecosistemas, de acuerdo con las normas sobre la materia.

(13) De acuerdo con el artículo 31 de la LGA, los ECA son "la[s] medida[s] que establece[n] el nivel de concentración o del grado de elementos, sustancias o parámetros físicos, químicos y biológicos, presentes en el aire, agua o suelo, en su condición de cuerpo receptor, que no representa riesgo significativo para la salud de las personas ni al ambiente". Así, los ECA son "obligatorio[s] en el diseño de las normas legales y las políticas públicas. [Son] un referente obligatorio en el diseño y aplicación de todos los instrumentos de gestión ambiental".

(14) De acuerdo con los considerandos del D.S. 038-2003.

(15) De acuerdo con lo dispuesto en los artículos 2 y 3 de la Ley SEIA. 
al SEIA, categorizados considerando el nivel de impacto ambiental negativo a evaluar:

Artículo 11.- Instrumentos de Gestión Ambiental del SEIA

Los instrumentos de gestión ambiental o estudios ambientales de aplicación del SEIA son:

a) La Declaración de Impacto Ambiental - DIA (Categoría I).

b) El Estudio de Impacto Ambiental Semidetallado - EIA-sd (Categoría II).

c) El Estudios de Impacto Ambiental Detallado - EIA-d (Categoría III).

d) La Evaluación Ambiental Estratégica - EAE.

Entiéndase para efectos del presente Reglamento, que las referencias a los estudios ambientales o los instrumentos de gestión ambiental comprenden indistintamente los señalados en este numeral (énfasis agregado).

Así, tenemos que los IGA sujetos al SEIA son: (i) la DIA, dirigido a evaluar proyectos de inversión que podrían generar impactos ambientales negativos leves (i.e. Categoría I); (ii) el EIAsd, dirigido a evaluar proyectos de inversión que podrían generar impactos ambientales negativos moderados (i.e. Categoría II); $y$, (iii) el EIA-d, dirigido a evaluar proyectos de inversión que podrían generar impactos ambientales negativos altos (i.e. Categoría III). Y, como el mismo artículo 11 del Reglamento SEIA lo indica en su último párrafo, estos son los IGA sobre los que se enfoca la regulación de la Ley SEIA y su reglamento.

Sin perjuicio de lo anterior, el artículo 13 del Reglamento SEIA destaca que los IGA no comprendidos en el SEIA son complementarios al mismo. Por ello, determina que:

[l]as obligaciones que se establezcan en dichos instrumentos deben ser determinadas de forma concordante con los objetivos, principios y criterios que se señalan en la Ley y el presente Reglamento, bajo un enfoque de integralidad y complementariedad de tal forma que se adopten medidas eficaces para proteger $y$ mejorar la salud de las personas, la calidad ambiental, conservar la diversidad biológica y propiciar el desarrollo sostenible, en sus múltiples dimensiones (énfasis agregado).

De manera que, y aunque sea evidente, los IGA complementarios al SEIA son todos aquellos que no forman parte de dicho sistema (i.e. DIA, EIA-sd y EIA-d) y que fueron aprobados a lo largo del tiempo, y siguen siendo aprobados, por los sectores correspondientes, a fin de prevenir, mitigar, remediar o adecuar los impactos de los proyectos que se pretendan ejecutar en el país; tales como los Programas de Adecuación y Manejo Ambiental, los Informes Técnicos Sustentatorios, Fichas Técnicas Ambientales, entre otros.

En este contexto, resulta crucial destacar que no todos los proyectos de inversión requieren de la aprobación de un IGA sujeto al SEIA para dar inicio a sus actividades. De hecho, el artículo 20 del Reglamento SEIA precisa que el SEIA "está orientado a la evaluación de los proyectos de inversión públicos, privados o de capital mixto, que por su naturaleza pudieran generar impactos ambientales negativos de carácter significativo (...)" (énfasis agregado). Si bien la norma no define qué se debe considerar como un impacto de carácter significativo, en el Anexo II del Reglamento del SEIA, el Ministerio del Ambiente ("MINAM") determinó expresamente que los proyectos de inversión sujetos al SEIA se encuentran dentro del "Listado de Inclusión de los Proyectos de Inversión sujetos al SEIA"; el cual es revisado y actualizado periódicamente, a fin de incluir nuevos proyectos dentro del sistema ${ }^{(16)}$.

Así, tenemos que los proyectos que no forman parte del SEIA están eximidos de tramitar y obtener la aprobación de un IGA preventivo (i.e. DIA, EIA-sd, EIA-d), a fin de dar inicio a sus actividades. No obstante lo anterior, de acuerdo con el artículo 23 del Reglamento del SEIA, "Ios proyectos, actividades, obras y demás que no están comprendidos en el SEIA deben ser desarrollados de conformidad con el marco legal vigente, debiendo el titular de los mismos cumplir todas las normas generales emitidas para el manejo de residuos sólidos, aguas, efluentes, emisiones, ruidos, suelos, conservación del patrimonio natural y cultural, zonificación, construcción y otros que pudieran corresponder" (énfasis agregado).

De manera que los titulares de los proyectos de inversión no incluidos en el SEIA, tienen la obligación de realizar sus actividades en cumplimiento las normas ambientales generales, emitidas a fin de evitar la generación de impactos ambientales negativos en el medio ambiente, los recursos naturales y la salud de las personas. Al respecto, es importante destacar que estas obligaciones no solo podrían derivar de las normas ambientales generales, sino también de Ios IGA complementarios que tanto el MINAM como el sector correspondiente

(16) La primera actualización del Anexo II del Reglamento SEIA fue en el 2011 con la Resolución Ministerial 157-2011-MINAM; y, la última modificación del listado fue en el 2020, con la Resolución Ministerial 023-2020-MINAM. 
podrían exigir tramitar a los titulares de los proyectos de inversión, ya sea antes de dar inicio a sus actividades, antes de modificar ciertos componentes de estas o, inclusive, a fin de adecuar ciertos aspectos de sus actividades a las normas ambientales vigentes.

Ahora, una vez que esta diferencia ha quedado clara, creemos que es lógico preguntarnos por qué no todos los proyectos de inversión deben estar sujetos al SEIA. Y esto se debe a que no todas las actividades económicas podrían generar impactos ambientales negativos significativos. De manera que no es factible exigir que un titular de un proyecto de inversión elabore y tramite un IGA sujeto al SEIA, considerando que, en la práctica, no se ha evidenciado que los efectos de dicha actividad podrían afectar de manera irreversible al medio ambiente, generando un daño irreparable en los recursos naturales y la salud de las personas; tal y como sí ha sucedido para el caso de los proyectos que forman parte del listado, aprobado en el Anexo II del Reglamento SEIA, y sus correspondientes actualizaciones.

Así, el contar con un listado que determine qué proyectos requieren o no, de la aprobación de un IGA preventivo sujeto al SEIA antes del inicio de sus actividades, genera seguridad jurídica entre los administrados. Lo anterior, toda vez que aquellos proyectos que no se encuentren dentro del listado, en principio, están habilitados a iniciar sus actividades sin necesidad de la previa aprobación de un IGA. Ello, considerando que, algunas veces, como lo hemos mencionados en los párrafos anteriores, el MINAM o el mismo sector exige que los titulares de proyectos de inversión no sujetos al SEIA, presenten un IGA complementario antes del inicio de sus actividades, tal y como sucede en el sector telecomunicaciones con el IGA denominado "Ficha Técnica Ambiental".

\subsubsection{Reglamentos de Protección Ambiental}

Finalmente, resulta importante mencionar que las obligaciones ambientales exigibles a los titulares de proyectos de inversión, no solo se encuentran estipuladas en las correspondientes normas ambientales generales; sino también en los denominados reglamentos de protección ambiental. Estos dispositivos normativos se aprobaron desde $1993^{(17)}$, sobre la base de lo dispuesto en el Código del Medio Ambiente y los Recursos Naturales, aprobado por Decreto Legislativo
$613^{(18)}$, norma que estableció los pilares de las principales obligaciones ambientales exigibles a los titulares de todo proyecto de inversión que podría generar algún tipo de impacto ambiental negativo en el medio ambiente y/o los recursos naturales; así como el artículo 50 de la Ley Marco para la Inversión Privada(19), aprobada por Decreto Legislativo $757^{(20)}$ que, entre otros, determinó que los ministerios eran las autoridades competentes para conocer los asuntos relacionados con la aplicación de las disposiciones del Código del Medio Ambiente y los Recursos Naturales.

Precisamente, los reglamentos de protección ambiental se aprobaron con el objetivo de que cada sector cuente con una norma específica que determine cuáles deberían ser los requisitos a cumplir por los administrados, a fin de que la ejecución de sus respectivos proyectos de inversión no genere impactos ambientales negativos en el medio ambiente, los recursos naturales y la salud de las personas. Por ello, es que a partir de la aprobación de los Decretos Legislativos 613 y 757 es que cada ministerio se vio en la obligación de publicar un reglamento que ponga en práctica, dentro de su sector correspondiente, las exigencias de las nuevas normas ambientales vigentes.

Esta práctica otorga seguridad jurídica a los administrados, toda vez que permite determinar con certeza cuáles son las características que debe acreditar un proyecto de inversión, con el objetivo de que el Estado permita su ejecución. Práctica que, de hecho, fue cumplida, dentro de un plazo razonable, por casi todos los ministerios. De hecho, la excepción a este actuar fue el MTC que, como veremos a continuación, a la fecha no cuenta con un reglamento de protección ambiental para el sector telecomunicaciones.

(17) El primer reglamento de protección ambiental aprobado en el ordenamiento jurídico nacional fue el del sector minero, mediante Decreto Supremo 016-93-EM.

(18) Publicado el 8 de septiembre de 1990.

(19) Artículo 50.- Las autoridades sectoriales competentes para conocer sobre los asuntos relacionados con la aplicación de las disposiciones del Código del Medio Ambiente y los Recursos Naturales son los Ministerios o los organismos fiscalizadores, según sea el caso, de los sectores correspondientes a las actividades que desarrollan las empresas sin perjuicio de las atribuciones que correspondan a los Gobiernos Regionales y Locales conforme a lo dispuesto en la Constitución Política.

(20) Publicado el 13 de noviembre de 1991. 


\section{Exigencias ambientales para la implementación de infraestructura de servicios públicos de telecomunicaciones}

En línea con lo señalado en el acápite II anterior, la Ley 29022 y sus reglamentos (el D.S. 039-2007 y el D.S. 003-2015) exigen a los titulares de infraestructuras de telecomunicaciones el cumplimiento de ciertas obligaciones ambientales, tales como: (i) la observancia del Principio Precautorio; (ii) el cumplimiento de los LMP de RNI; y, (iii) la obtención de un IGA (sujetos y complementarios al SEIA).

Sin perjuicio de estas exigencias, destacamos también la necesidad de que el sector telecomunicaciones apruebe un reglamento de protección ambiental. En el marco de la elaboración del presente trabajo, hemos tomado conocimiento de que el MTC, en coordinación con el MINAM, se encuentra trabajando en un proyecto de reglamentación. Sin embargo, a la fecha, este aún no ha sido aprobado. A continuación, pasaremos a desarrollar cada uno de estos aspectos.

\subsection{EI Principio Precautorio y los LMP de RNI}

La Ley 29022, en concordancia con lo dispuesto en la LGA, exige a los administrados la observancia del Principio Precautorio para la instalación y despliegue de infraestructura de telecomunicaciones. En línea con ello, el artículo 10 de la referida ley ${ }^{(21)}$ establece que las autoridades administrativas tienen la obligación de supervisar la aplicación y el cumplimiento de este principio durante el desarrollo de estas actividades.

Al respecto, resulta importante destacar que la normativa sectorial ha determinado que "la aplicación del Principio Precautorio implica el cumplimiento de los LMP de RNI"(22). En ese sentido, tanto el artículo 5 del D.S. 039-2007 (derogado) como el artículo 6 del D.S. 003-2015 (actualmente vigente) establecen lo siguiente:

El Principio Precautorio o Principio de Precaución a que se refiere el literal e) del artículo 2 de la Ley, se encuentra referido al cumplimiento de los Límites Máximos Permisibles de Radiaciones No lonizantes en Telecomunicaciones aprobados por Decreto Supremo 038-2003-MTC y su modificatoria. Para tal efecto, a fin de fiscalizar su efectivo cumplimiento, el Ministerio deberá observar las disposiciones contenidas en el artículo 11 de la Ley 28611, Ley General del Ambiente, el citado Decreto Supremo 038-2003-MTC y su modificatoria, así como las Normas Técnicas sobre Restricciones Radioeléctricas en áreas de Uso Público, aprobadas mediante Resolución Ministerial 120-2005-MTC-03 (énfasis agregado).

De esa manera, los solicitantes y titulares de concesiones o autorizaciones de telecomunicaciones deben respetar los LMP de RNI establecidos en el artículo 3 del D.S. 038-2003. Con la finalidad de garantizar el cumplimiento de esta disposición, la norma señala que los titulares y solicitantes, según corresponda, deben presentar estudios teóricos y realizar monitoreos a sus infraestructuras, de conformidad con los lineamientos, parámetros, entre otros que sean aplicables, regulados en el D.S. 0382003 y en las normas complementarias que dicte el MTC sobre esta materia. Así, el D.S. 038-2003 busca evitar la transgresión de los LMP y, por tanto, que las RNI no generen impactos negativos o perjudiciales en la salud de las personas y/o en el medio ambiente.

Considerando que el objetivo del D.S. 038-2003 es impedir la configuración de un posible daño como consecuencia de la vulneración de los LMP de RNI, se podría inferir que su cumplimiento encontraría mayor relación con la aplicación del Principio de Prevención, recogido en el artículo VI del Título Preliminar de la LGA. Sin embargo, desde que entró en vigencia el régimen especial implementado por la Ley 29022 (i.e. 2007), el MTC decidió circunscribir el cumplimiento de estos LMP al Principio Precautorio, sin que ello haya sido materia de discusión en las modificatorias de esta ley y/o en algunos de sus reglamentos.

Una posible explicación a esta vinculación la podemos encontrar en la Exposición de Motivos del D.S. 039-2007, que aprobó el primer reglamento de la Ley 29022. En este documento se indica que el MTC habría tomado la decisión de aprobar los LMP de RNI, debido a que el Tribunal Constitucional invocó el Principio Precautorio en la sentencia que resolvió una demanda de amparo

(21) Artículo 10.- Cumplimiento de la Ley

Las entidades del Estado, en los ámbitos de sus respectivas competencias, supervisarán el fiel cumplimiento de la presente Ley, correspondiendo al Gobierno Nacional, a través de las instancias competentes, la aplicación y supervisión del principio de precaución en materia de instalación y despliegue de Infraestructura Necesaria para la Prestación de Servicios Públicos de Telecomunicaciones. (...).

(22) El Anexo I del D.S. 038-2003 recoge la siguiente definición de RNI: "Es la que no produce ionización en la materia. Cuando atraviesa los tejidos vivos, no tiene la suficiente energía para dañar el ADN en forma directa." 
interpuesta en contra de la empresa Nextel del Perú S.A.C. por la instalación de una antena y equipos destinados a la prestación del servicio de telefonía móvil:

Como consecuencia de la Sentencia del Tribunal Constitucional recaída en el Expediente 0964-2002-AA/TC, de 17 de marzo del 2003, que invocó el Principio de Precaución o Precautorio, el Ministerio de Transportes y Comunicaciones, mediante Decreto Supremo 038-2003-MTC, del 3 de julio del 2003 ha establecido los Límites Máximos Permisibles de Radiaciones no lonizantes en Telecomunicaciones, los que constituyen un instrumento de gestión ambiental prioritario para prevenir y controlar la contaminación generado por actividades de telecomunicaciones sobre la base de una estrategia destinada a proteger la salud, mejorar la competitividad del país y promover el desarrollo sostenible" (énfasis agregado).

Sin embargo, de una revisión del D.S. 038-2003 y su Exposición de Motivos no encontramos referencia alguna a dicho pronunciamiento del Tribunal Constitucional y/o al Principio Precautorio. Por el contrario, la Exposición de Motivos del D.S. 038-2003 señala que la importancia de esta norma radicaría, entre otros, en que los LMP de RNI: (i) constituyen una valiosa herramienta de gestión que permitiría un manejo adecuado de las expectativas de los actores involucrados en el sector; (ii) revaloran el tema ambiental como un asunto de interés público, económico y político; (iii) son una oportunidad para corregir deficiencias generadas por el vacío normativo respecto a este asunto; y, (iv) reafirman la necesidad de establecer la vigencia de códigos de conducta empresarial en términos económicos y de bienestar social.

Sin perjuicio de ello, cabe destacar que la sentencia recaída en el Expediente 0964-2002-AA/TC constituye uno de los primeros pronunciamientos en los que el Tribunal Constitucional incluyó el Principio Precautorio en su análisis respecto de la instalación de infraestructura de telecomunicaciones. No obstante, ello, según indica Ramón Huapaya, es importante anotar que en dicha oportunidad este principio habría sido introducido como un "elemento auxiliar" en la argumentación del Tribunal Constitucional, que finalmente concluyó que la empresa demandada debía retirar la antena y los equipos instalados (2011, p. 749).

Si bien la sentencia se enfoca en analizar con mayor detalle la posible afectación del derecho a un ambiente equilibrado y adecuado, el Tribunal Constitucional señala también que, al amparo del Principio Precautorio, la normativa sectorial debe disponer la aplicación de determinadas medidas que tengan como finalidad evitar que este tipo de instalaciones sean desplegadas en zonas residenciales o de gran afluencia de personas (como hospitales o colegios):

11. Respecto de la posible afectación del derecho a la salud y a un medio ambiente sano y adecuado a consecuencia de la propagación de ondas electromagnéticas, este Tribunal debe destacar que se trata de un tema en el que, desde un punto de vista científico, no existe actualmente consenso. Sí existe consenso, sin embargo, en que a través de la legislación correspondiente se establezca una serie de precauciones destinadas a evitar que la carencia de resultados satisfactorios en la investigación sobre el tema, no termine generando problemas irreversibles en la salud y el medio ambiente, $y$, en ese sentido, que en la medida de lo posible tales equipos y antenas se instalen en lugares donde la gente no pase prolongados periodos de tiempo. Forma parte de ese denominado 'principio de precaución', que el Estado prevea a través de medidas de regulación en la prestación de ese servicio público o mediante la regulación de la materia urbanística, que la instalación de tales equipos y antenas no se efectúe cerca de hospitales, escuelas o zonas residenciales, y que se asegure que los que operan en el mercado en la prestación del servicio público en referencia, compartan torres para reducir su número (...) (énfasis agregado).

Considerando lo anterior, en este caso el Tribunal Constitucional habría aplicado una "versión fuerte" del Principio Precautorio. En líneas generales, la aplicación de una versión fuerte de este principio implica que, ante la incertidumbre científica respecto del daño que podría generar una actividad determinada, las autoridades deben adoptar medidas destinadas a prevenir o prohibir dicha actividad, por lo menos hasta que la evidencia científica determine que no se configurará un daño a la salud y/o al medio ambiente ${ }^{(23)}$.

(23) Al respecto, Ramón Huapaya señala que: “(...) el principio de precaución conoce dos versiones en la legislación internacional y la literatura medio ambiental: una 'débil' y una 'fuerte'. La versión débil consiste en la falta de evidencia decisiva para la producción de un daño no debe ser un obstáculo o impedimento para regular la situación riesgosa. Por otro lado, la versión 'fuerte', conocer muchas variantes, siendo la más intervencionista, aquélla que establece que cuando la realización de una actividad humana produzca o pueda producir el riesgo de un daño significativo para el ambiente o para la salud de la colectividad o de las futuras generaciones, $y$ exista incerteza científica sobre la naturaleza y entidad del daño o riesgo que pueda causárseles, entonces deba tomarse decisiones para prevenir o prohibir la realización de tales actividades, al menos hasta que la evidencia científica demuestre que el daño no ocurrirá" (2011, p. 746). 
Posteriormente, el Tribunal Constitucional modificó su criterio respecto de la aplicación de este principio. Así, si bien el supremo intérprete de la Constitución reconoce que para la aplicación del Principio Precautorio debe existir falta de certeza científica sobre los daños que se puedan ocasionar a la salud y/o al medio ambiente, es necesario que se presenten indicios razonables y suficientes que justifiquen la adopción de medidas al respecto. En particular, en la sentencia recaída en el Expediente 4223-2006-PA/TC se indica lo siguiente:

(...) el principio precautorio se aplica ante la amenaza de un daño a la salud o medio ambiente y ante la falta de certeza científica sobre sus causas y efectos. Si bien el presupuesto esencial para la aplicación del principio precautorio es precisamente la falta de certeza científica -aun cuando no sea imprescindible demostrar plenamente la gravedad y la realidad del riesgo-, sí resulta exigible que haya indicios razonables y suficientes de su existencia y que su entidad justifique la necesidad de adoptar medidas urgentes, proporcionales y razonables (énfasis agregado).

De esa forma, el Tribunal Constitucional reconoció que no siempre la prohibición absoluta de una actividad es la única vía para alcanzar determinado grado de protección, este podrá ser alcanzado mediante la reducción de la exposición al riesgo, con el establecimiento de mayores controles y la imposición de ciertas limitaciones.

\subsection{Los IGA en el sector telecomunicaciones}

Considerando las exigencias del Reglamento SEIA, el 21 de julio de 2011, mediante la Resolución Ministerial 157-2011-MINAM, el MINAM aprobó la primera actualización del Listado de Inclusión de los Proyectos de Inversión sujetos al SEIA ("Listado"). Así, respecto del sector telecomunicaciones, el Listado indicó que se encontraba sujeto al SEIA, todo proyecto de inversión dirigido a la instalación y operación de infraestructura de telecomunicaciones; lo cual incluía las redes o infraestructuras de telecomunicaciones, sean éstas inalámbricas o radioeléctricas, como alámbricas o por cable.

En ese sentido, todos los proyectos del sector que cumplieran con dichas características, tenían la obligación de tramitar ante el MTC, la obtención de un IGA sujeto al SEIA (i.e. DIA, EIA-sd o EIA-d), antes del inicio de sus actividades. No obstante lo anterior, y sobre la base de lo dispuesto en el artículo 20 del Reglamento SEIA ${ }^{(24)}$, en el 2015 el Listado fue actualizado, y se amplió el ámbito de aplicación del SEIA a los proyectos del sector comunicaciones.

Precisamente, el 1 de agosto de 2015, con la aprobación de la Resolución Ministerial 186-2015-MINAM ("RM 1862015 "), se incluyó dentro del SEIA a los proyectos del sector telecomunicaciones con las siguientes características, como lo establece el artículo 1 de la RM 186-2015:

- Proyectos de Infraestructura de telecomunicaciones que incluye las redes o infraestructuras de telecomunicaciones, sean éstas inalámbricas o radioeléctricas, como alámbricas o por cable;

- Para el caso de proyectos de infraestructuras de servicios móviles (servicio de telefonía móvil, fija e internet) tipo poste se incluyó en el Listado aquellas que cumplen con alguno de los siguientes criterios:

a) Que sus características no estén contempladas en las opciones de mimetización establecidas en el D.S. 003-2015.

b) Que se localicen dentro de Áreas Naturales Protegidas o en sus zonas de amortiguamiento o dentro de ecosistemas frágiles, en cumplimiento de la legislación de la materia.

c) Que el valor del cálculo teórico de RNI de la infraestructura de telecomunicaciones supere el $50 \%$ del valor establecido como LMP de RNI en Telecomunicaciones para la exposición ocupacional o exposición poblacional, de acuerdo al D.S. 038-2003.

- Para el caso de proyectos de infraestructuras de servicios móviles (servicio de telefonía móvil, fija e internet) tipo rooftop se incluyó en el Listado aquellas que cumplen con alguno de los siguientes criterios:

a) Que sus características no estén contempladas en las opciones de mimetización establecidas en el D.S. 003-2015.

b) Que la altura del soporte y antena en su conjunto supera $2 / 3$ de la altura de la edificación.

c) Que se localicen dentro de Áreas Naturales Protegidas o en sus zonas de amortiguamiento o dentro de ecosistemas frágiles, en cumplimiento de la legislación de la materia.

(24) Artículo 20.- Proyectos de inversión que están sujetos al SEIA

(...) Los proyectos que comprende el SEIA se encuentran señalados en el Listado de Inclusión de los Proyectos de Inversión sujetos al SEIA previsto en el Anexo II. EI MINAM revisa y actualiza periódicamente este Listado en coordinación con las entidades que conforman el SEIA (énfasis agregado). 
d) Que el valor del cálculo teórico de RNI de la infraestructura de telecomunicaciones supere el $50 \%$ del valor establecido como LMP de RNI en Telecomunicaciones para la exposición ocupacional o exposición poblacional, de acuerdo al D.S. 038-2003.

- Para el caso de proyectos de infraestructuras de servicios móviles (servicio de telefonía móvil, fija e internet) tipo greenfield se incluyó en el Listado aquellas que cumplen con alguno de los siguientes criterios:

a) Que la altura del soporte y antena en su conjunto supera los 30 metros y que se localicen dentro de área urbana o expansión urbana.

b) Que se localicen dentro de Áreas Naturales Protegidas o en sus zonas de amortiguamiento o dentro de ecosistemas frágiles, en cumplimiento de la legislación de la materia.

c) Que el valor del cálculo teórico de RNI de la infraestructura de telecomunicaciones supere el $50 \%$ del valor establecido como LMP de RNI en Telecomunicaciones para la exposición ocupacional o exposición poblacional, de acuerdo al D.S. 038-2003.

Sin embargo, el artículo 2 de la resolución ministerial en análisis, reguló la figura de la Ficha Técnica Ambiental ("FTA"), IGA complementario al SEIA, exigible a todos aquellos titulares de proyectos de inversión del sector telecomunicaciones que no se encuentren dentro del SEIA. De hecho, en dicho artículo se establece que la presentación de esta FTA es requisito indispensable para obtener la Autorización para la Instalación de Infraestructura de Telecomunicaciones, considerando lo dispuesto en el literal f) del artículo 12 del D.S. 003-2015(25).

En ese sentido, tenemos que los titulares de proyectos del sector telecomunicaciones podrían encontrarse obligados a tramitar para el inicio de ejecución de sus proyectos: (i) IGA sujetos al SEIA: DIA, EIA-sd y EIA-d; y/o, (ii) IGA complementarios al SEIA: FTA; lo anterior, tomando en cuenta el nivel del impacto de sus actividades. En otras palabras:

Corresponde un IGA sujeto al SEIA: Cuando el proyecto de inversión del sector telecomunicaciones podría generar impactos ambientales negativos leves, moderados o altos. Que, de acuerdo con la RM 186-2015, podrían ser las siguientes infraestructuras:

- De infraestructura de telecomunicaciones que incluye las redes o infraestructuras de telecomunicaciones, sean estas inalámbricas o radioeléctricas, como alámbricas o por cable.
- De infraestructuras de servicios móviles (servicio de telefonía móvil, fija e internet) tipo poste, siempre que cumplan con ciertos criterios.

- De infraestructuras de servicios móviles (servicio de telefonía móvil, fija e internet) tipo rooftop, siempre que cumplan con ciertos criterios.

- De infraestructuras de servicios móviles (servicio de telefonía móvil, fija e internet) tipo greenfield, siempre que cumplan con ciertos criterios.

Corresponde un IGA complementario al SEIA: Cuando el proyecto de inversión no prevea la generación de impactos ambientales negativos $y$, por tanto, no se encuentre dentro del Listado (i.e. FTA).

Adicionalmente, destacamos que el 7 de mayo de 2021 se publicó la Resolución Ministerial 076-2021-MINAM mediante la cual se modificó el Listado a fin de incluir nuevos proyectos de inversión del sector telecomunicaciones en el SEIA, tales como proyectos de infraestructura para la prestación de servicios inalámbricos (servicios de voz, datos y videos tanto fijos como móviles) tipo poste, tipo rooftop y tipo greenfield que cumplan con determinadas características relacionadas a su ubicación dentro de áreas naturales protegidas de administración nacional, áreas de conservación nacional o cuyas características no estén contempladas en la opciones de mimetización establecidas en la normativa correspondiente.

Sobre el particular, y tomando en cuenta la información brindada por la Dirección General de Políticas y Regulación en Comunicaciones del MTC ${ }^{(26)}$, resulta importante destacar que entre el 2019 y el 2020 se aprobaron un total de $182^{(27)}$ DIA en el MTC. Considerando que este fue el único IGA solicitado y aprobado por los titulares de los proyectos del sector. Y, por otro lado, entre el mismo periodo de años, el MTC otorgó la

(25) Artículo 12.- Requisitos Generales para la Aprobación Automática de una Autorización

Los Solicitantes de una Autorización presentan a la Entidad competente los siguientes documentos: (...)

f. Instrumento de gestión ambiental aprobado por el Ministerio.

(26) Solicitada a través del procedimiento de acceso a la información pública.

(27) De los cuales, (i) 106 corresponden al 2019 y (ii) 76 corresponden al 2020. 
conformidad a 11,436 FTA ${ }^{(28)}$. De lo anterior, se desprende que existen muchos más proyectos del sector telecomunicaciones que se ejecutan sin la previa aprobación de un IGA sujeto al SEIA; y, por tanto, aparentemente no deberían generar impactos ambientales negativos significativos.

En este contexto, destacamos que el hecho de no encontrarse sujeto al SEIA no constituye un eximente al cumplimiento de las normas ambientales generales y especiales del sector telecomunicaciones; dentro de las que se encuentran el Principio Precautorio, los LMP de RNI y los ECA para RNI. De hecho, lo anterior se deriva de lo dispuesto en el artículo 23 del Reglamento SEIA, por el cual expresamente se indica que:

Artículo 23.- Proyectos, actividades, obras y otros no comprendidos en el SEIA

Sin perjuicio de lo señalado en el artículo precedente y de las normas especiales que se emitan, los proyectos, actividades. obras y demás que no están comprendidos en el SEIA deben ser desarrollados de conformidad con el marco legal vigente, debiendo el titular de los mismos cumplir todas las normas generales emitidas para el manejo de residuos sólidos, aguas, efluentes, emisiones, ruidos, suelos, conservación del patrimonio natural y cultural, zonificación, construcción y otros que pudieran corresponder (énfasis agregado).

En esa línea, el artículo 3 de la RM 186-2015, de forma complementaria a lo dispuesto en el artículo 23 citado, establece lo siguiente con relación a la exigencia de cumplir con los LMP y ECA para RNI:

Artículo 3.- Los operadores de servicios móviles cuyas infraestructuras se encuentren o no sujetas al Sistema Nacional de Evaluación de Impacto Ambiental (SEIA) deberán cumplir con los monitoreos y mediciones periódicas de Radiaciones No lonizantes, de conformidad con las disposiciones emitidas para el control de Límites Máximos Permisibles y Estándares de Calidad Ambiental y demás normativas vigentes (énfasis agregado).

Adicionalmente, el numeral 6 del formato general de la FTA, aprobado por la RM 186-2015, exige que el titular declare lo siguiente:

6. CUMPLIMIENTO DE LOS ESTÁNDARES DE CALIDAD AMBIENTAL Y LÍMITES MÁXIMOS PERMISIBLES DE RADIACIONES NO IONIZANTES (RNI)

Declaro que el presente proyecto de infraestructura de telecomunicaciones cumple con los Estándares de Calidad Ambiental y Límites Máximos Permisibles de RNI establecidos en la legislación y que se realizarán los monitoreos regulares para verificar el mencionado desempeño (énfasis agregado).

Así pues, es una exigencia general para todos los titulares de infraestructura de telecomunicaciones realizar monitoreos ambientales a fin de verificar el cumplimiento de los ECA y LMP de RNI. Los ECA, de acuerdo con el artículo 31 de la LGA, se definen de la siguiente manera:

Artículo 31.- Del Estándar de Calidad Ambiental

31.1 El Estándar de Calidad Ambiental ECA es la medida que establece el nivel de concentración o del grado de elementos. sustancias o parámetros físicos, químicos y biológicos, presentes en el aire, agua o suelo, en su condición de cuerpo receptor, que no representa riesgo significativo para la salud de las personas ni al ambiente. Según el parámetro en particular a que se refiera, la concentración o grado podrá ser expresada en máximos, mínimos o rangos (énfasis agregado).

Así pues, a diferencia de los LMP, los ECA analizan las consecuencias de las $\mathrm{RNI}$ en el cuerpo receptor (por ejemplo, los seres vivos). $\mathrm{Y}$, sobre lo anterior, se regulan valores de $\mathrm{RNI}$ que todo titular de un proyecto de infraestructura de telecomunicaciones debe respetar durante la ejecución de sus actividades, a fin de evitar la generación de impactos negativos al medio ambiente, los recursos naturales y la salud de las personas.

En línea con lo anterior, de forma adicional a la aprobación de los LMP para RNI, el MINAM aprobó los ECA para RNI, mediante Decreto Supremo 010-2005-PCM ("D.S. 010-2005"); y, posteriormente, mediante Resolución Ministerial 218-2020-MINAM se aprobaron los "Lineamientos para la elaboración de planes de acción para la prevención y control de RNI generadas por los servicios de telecomunicaciones y las redes eléctricas". Este último con el objetivo de orientar a las municipalidades provinciales en la elaboración de los planes de acción para la prevención y control de las RNI en sus respectivas jurisdicciones, considerando lo dispuesto en el artículo 8 del D.S. 010-2005.

Así las cosas, queda claro que los titulares de infraestructuras de telecomunicaciones tienen el deber de obtener la aprobación de un IGA, antes del inicio de sus actividades, siempre que se encuentren sujetos al SEIA;

(28) De los cuales, (i) 9854 corresponden al 2019 y (ii) 1582 corresponden al 2020. 
es decir, cuando su proyecto se subsuma en alguno de los supuestos de la RM 186-2015. Y, por otro lado, aquellos proyectos que no se encuentren sujetos al SEIA, solo tiene la obligación de presentar una FTA antes del inicio de sus actividades; deber que por la pandemia generada a causa de la propagación del COVID-19, ha sido "suspendido", de acuerdo con el artículo 9 del Decreto Legislativo $1477^{(29)}$.

No obstante, lo anterior, todos los titulares de proyectos de infraestructuras de telecomunicaciones se encuentran obligados a cumplir con los LMP y ECA para RNI durante la ejecución de sus actividades. Como hemos demostrado a lo largo del presente artículo, el cumplimiento de estos parámetros resulta esencial, toda vez que la mayoría de los proyectos del sector no requieren de la previa aprobación de un IGA antes de su inicio. Por ello, finalmente, la única forma de prevenir cualquier tipo de impacto de esta actividad sobre el medio ambiente, los recursos naturales y la salud de las personas, es enfatizar en el cumplimiento de las normas ambientales generales del sector; es decir, los LMP de RNI y los ECA para RNI.

\subsection{El Reglamento de Protección Ambiental del sector telecomunicaciones}

Como adelantamos en el numeral II anterior, el sector telecomunicaciones no cuenta, ni ha contado, con un reglamento de protección ambiental que determine cuáles son las obligaciones ambientales o los requisitos específicos que un administrado debe cumplir y acreditar, a fin de evitar que el desarrollo de su actividad no genere impactos ambientales negativos en el medio ambiente, los recursos naturales y la salud de las personas. De hecho, con relación a la materia ambiental, el MTC solo ha cumplido con aprobar un reglamento de protección ambiental para las actividades relacionadas con el sector transportes.

Sin embargo, y como hemos mencionado en el apartado b) anterior, desde el 2003, con el D.S. 038-2003 que aprobó los LMP de RNI, luego en el 2005 con la aprobación del D.S. 010-2005, relacionado con los ECA para RNI y, posteriormente, con la aprobación de la RM 186-2015; el MINAM y el MTC han determinado la normativa ambiental base para el sector telecomunicaciones, que todo titular de un proyecto del sector debe tomar en cuenta de forma previa al inicio de sus actividades. De manera que, y aun cuando no exista un reglamento de protección ambiental específico del sector, queda claro que los titulares de proyectos de infraestructura de telecomunicaciones, no están eximidos del cumplimiento de disposiciones ambientales, antes y durante de la ejecución de sus actividades.

A pesar de lo anterior, consideramos que es necesario que el MTC apruebe, cuanto antes, un reglamento de protección ambiental que compile en un solo cuerpo normativo las obligaciones ambientales exigibles a los proyectos de infraestructura del sector telecomunicaciones. Así, se agilizaría la evaluación de los permisos ambientales del sector, antes de plantear la ejecución de actividades de telecomunicaciones en el país; y, ello, permitiría un adecuado cumplimiento y fiscalización de las obligaciones ambientales correspondientes.

Lo anterior, inclusive, tendría que ir de la mano con una modificación del Reglamento de Organización y Funciones del MTC, a fin de que se establezca quién será la dirección encargada de normar, evaluar, fiscalizar y sancionar el cumplimiento de las obligaciones ambientales que, actualmente, estarían siendo asumidas, parcialmente, por la Dirección General de Políticas y Regulación en Comunicaciones ${ }^{(30)}$. Claro está, mientras dichas funciones no sean transferidas al $\mathrm{OEFA}^{(31)}$ (i.e. en materia de fiscalización y sanción ambiental) o al SENACE(32) (i.e. en materia de evaluación ambiental).

(29) Artículo 9. Disposiciones ambientales para los proyectos de Infraestructura de Telecomunicaciones no comprendidos en el Sistema de Evaluación de Impacto Ambiental

9.1 En tanto dure la Emergencia Sanitaria a nivel nacional declarada por Decreto Supremo 008-2020-SA y/o ampliatorias, se exonera a los operadores de los servicios públicos de telecomunicaciones y proveedores de infraestructura pasiva de la obligación de presentar la Ficha Técnica regulada por el artículo 2 de la Resolución Ministerial 186-2015-MINAM, respecto a los proyectos de infraestructura de telecomunicaciones que no están sujetos al Sistema de Evaluación de Impacto Ambiental (SEIA).

(30) Artículo 149 del Texto Integrado del Reglamento de Organización y Funciones del MTC, aprobado por Resolución Ministerial 07852020-MTC/01.

(31) De acuerdo con lo dispuesto en la Primera Disposición Complementaria Final y en la Primera Disposición Complementaria Transitoria de la Ley del Sistema Nacional de Evaluación y Fiscalización Ambiental, aprobada por Ley 29325.

(32) Considerando lo dispuesto en el Cronograma de Transferencia de Funciones de las Autoridades Sectoriales al servicio del SENACE, aprobado por Decreto Supremo 006-2015-MINAM. 


\section{Conclusiones y recomendaciones}

Como hemos podido apreciar en el presente artículo, el sector telecomunicaciones cuenta con disposiciones normativas que exigen el cumplimiento de obligaciones y parámetros ambientales, a los titulares de proyectos de infraestructura, tanto antes como durante la ejecución de sus actividades. Ello, con el objetivo de evitar que estos proyectos generen impactos ambientales negativos en el medio ambiente, los recursos naturales y la salud de las personas. De hecho, y considerando las implicancias del Principio Precautorio, el MTC, junto con el MINAM, determinaron promover los proyectos del sector telecomunicaciones, requiriendo acreditar el cumplimiento de los LMP de RNI, así como los ECA para RNI. Lo anterior, considerando que las actividades del sector no generarían impactos ambientales negativos que requieran de la tramitación de un IGA.

No obstante, con el paso de los años y el consecuente desarrollo científico respecto de los posibles impactos ambientales negativos significativos que podrían generar los proyectos del sector, se aprobó un listado de proyectos que requerían de la aprobación de una IGA sujeto al SEIA, antes del inicio de sus actividades. Y, a partir de dicho momento, fue necesario para los titulares de proyectos de infraestructura, identificar cómo calificaría su proyecto dentro del sistema ambiental; toda vez que ello implicaría la tramitación de permisos adicionales para dar inicio a sus actividades, tal y como la elaboración y posterior aprobación de un IGA.

Si bien, lo anterior, fue un avance significativo del sector en materia ambiental, resulta necesario contar con un reglamento de protección ambiental que detalle todas las obligaciones ambientales generales y específicas del sector telecomunicaciones y, de ser necesario, se remita a las disposiciones específicas del sector (i.e. LMP de RNI y ECA para $\mathrm{RNI}$ ). De hecho, según hemos indicado anteriormente, el MTC, en coordinación con el MINAM, se encontraría elaborando un proyecto de reglamentación. Sin embargo, a la fecha, este aún no ha sido aprobado. Resaltamos la importancia del rol del MINAM en este trabajo, toda vez que su participación permitirá que el futuro reglamento regule de manera correcta y ordene los mecanismos ambientales del sector.

Esto último otorgaría mayor predictibilidad a los inversionistas de proyectos de infraestructura de telecomunicaciones, toda vez que sería mucho más factible identificar en un solo cuerpo normativo: (i) los permisos requeridos para iniciar con este tipo de actividades en el país, así como (ii) las respectivas autoridades administrativas a cargo de la evaluación, fiscalización y sanción en materia ambiental del sector. Por ello es que, y a manera de recomendación, creemos que es vital que el MTC apruebe, cuanto antes, un reglamento de protección ambiental para el sector telecomunicaciones que vaya de la mano con un dispositivo que determine las infracciones y sanciones por el incumplimiento de las obligaciones ambientales. Lo anterior, como comentábamos, no sólo agilizará la toma de decisiones de los inversionistas; sino que, además, permitirá que este tipo de actividades en el país sea evaluado y fiscalizado de forma adecuada, evitando la generación de impactos ambientales negativos en el medio ambiente, los recursos naturales y la salud de las personas.

\section{Referencias bibliográficas}

Alegre, A. (2010). Sobre la relevancia del principio precautorio. Gaceta Constitucional, 27, 19-29.

De La Puente, L. (2008). La Industria y la Rigidez Actual en la Aplicación de los Límites Máximos Permisibles: Caben Excepciones. THEMIS Revista De Derecho, 56, 219-229.

Huapaya, R. (2011). Contra "las leyes del miedo": Un ensayo crítico sobre el principio de precaución y su aplicación en el Derecho Público. Administración Pública, Derecho Administrativo y Regulación. Estudios y cuestiones, 740-756. Ara Editores.

Tribunal Constitucional. (2003). Sentencia recaída en el Expediente 0964-2002-AA/TC.

Tribunal Constitucional. (2007). Sentencia recaída en el Expediente 4223-2006-PA/TC.

Zegarra, D. (2008). La instalación de infraestructuras para la prestación del servicio público de telecomunicaciones: los problemas en el ámbito municipal. El Derecho Administrativo y la modernización del Estado Peruano. Ponencias del Tercer Congreso Nacional de Derecho Administrativo. Grijley. (40) 